\title{
TRANSPLANTE RENAL PEDIÁTRICO E UROPATIA OBSTRUTIVA
}

\author{
Renal transplantation and obstructive uropathy
}

Rejane de Paula Meneses ${ }^{1}$, Cecilia Hallusch², Eliane Ribeiro Carmes ${ }^{6}$

\section{RESUMO}

Uropatia obstrutiva é causa freqüente de insuficiência renal crônica na infância. Protocolo clínico e cirúrgico que viabilize a reconstrução do trato urinário antes do transplante é fundamental no prognóstico do enxerto. Objetivo: comparar função e sobrevida do enxerto em pacientes com e sem uropatia e descrever a evolução daqueles com ampliação vesical. Método: estudadas 62 crianças transplantadas entre 1990 e 2000, no Hospital Pequeno Príncipe, Curitiba, Paraná, com mais de 6 meses de seguimento, 32 (51,6\%) com uropatia, todas submetidas à avaliação urológica antes e após o transplante. Resultados: $68 \%$ das crianças estudadas eram do sexo masculino, com idade de 12,1 \pm 4 anos (média \pm desvio-padrão). Não houve diferença significativa entre uropatas e não-uropatas em relação a sexo, idade, mortalidade, tipo de doador, compatibilidade, rejeição aguda e crônica, perda do enxerto e clearance de creatinina. Infecção do trato urinário ocorreu somente no grupo dos uropatas. Nesse grupo foi realizada nefrectomia unilateral pré-transplante em $8,6 \%$ dos pacientes e bilateral em 82,8\%; ampliação vesical em $37,1 \%$ e conduto para cateterismo em $45,7 \%$. O estudo urodinâmico evidenciou diferença significativa na capacidade e na complacência vesical antes e após a ampliação e após o transplante. As crianças com ampliação vesical não apresentaram maiores taxas de refluxo vésico-ureteral ou litíase mas apresentaram maior incidência de infecção do trato urinário e de hidronefrose. Conclusão: um protocolo clínico-cirúrgico diferenciado para crianças com uropatia, que contemple diagnóstico e correção no pré-transplante e a evolução no pós-transplante, está relacionado com uma evolução favorável e com bom prognóstico do enxerto.

Descritores: Transplante renal, Uropatia obstrutiva, Uropatia, Pediatria, Terapia de substituição renal.

Trabalho realizado no Hospital Pequeno Príncipe de Curitiba - Brasil Formação Acadêmica

${ }^{1}$ Nefropdiatra, chefe do Serviço de Nefrologia

${ }^{2}$ Nefropdiatra, responsável pela Unidade de Transplante Renal

${ }^{3}$ Assessora em Pesquisa Clínica, mestre em Medicina Interna Instituição: Hospital Pequeno Príncipe de Curitiba - Brasil Endereço para correspondência: Dra. Rejane de Paula Meneses Rua Petit Carneiro, 1111 - apto. 2201 - CEP 80240-050 - Curitiba - PR - Brasil Fone/fax (41) 3101225 - E-mail: rejanemeneses@onda.com.br Recebido em: 30/06/2003 Aceito em: 05/01/2003

\section{INTRODUÇÃO}

As alterações do trato urinário são responsáveis por 48,5\% dos casos de insuficência renal crônica na infância.1 Anomalias congênitas, como mielodisplasia, bexiga neurogênica, válvula de uretra posterior e disfunção vesical, resultam em bexigas de pequena capacidade, com pouca complacência e resíduo urinário que dependem de tratamento específico para recuperar a função do trato urinário inferior e garantir pressão de esvaziamento vesical adequada para proteção do enxerto. Uma bexiga normal funciona como reservatório urinário de baixa pressão, continente, estéril que se esvazia espontânea e completamente. Infecções de trato urinário com sepse e disfunção renal podem ocorrer quando estas funções encontram-se alteradas em uma bexiga nativa ou reconstruída. Procedimentos como construção de um conduto para cateterismo, com apêndice ou ureter, e ampliação vesical, com segmentos de estômago, intestino ou de ureter dilatado, têm sido realizados com índices decrescentes de complicações, atribuídos a avanços, ocorridos nas últimas duas décadas, na técnica cirúrgica, na imunossupressão e no manejo clínico. Assim crianças com uropatia consideradas não elegíveis para transplante ou como fator de risco para o enxert, podem 
hoje ser incluídas em programa de transplante após a correção ou reconstrução do trato urinário. Este estudo foi delineado para comparar características clínicas e demográficas em crianças uropatas e não-uropatas e para descrever a evolução das crianças com ampliação vesical.

\section{Pacientes e Métodos}

Entre 1990 e 2000, 67 pacientes foram submetidos a transplante renal no Hospital Pequeno Príncipe de Curitiba, Paraná. Sessenta e dois pacientes $(92,5 \%)$ com enxerto funcionante e com seguimento mínimo de 6 meses foram incluídos neste estudo de coorte histórica, $32(51,6 \%)$ com insuficiência renal crônica secundária a uropatia: bexiga neurogênica $(34,4 \%)$, refluxo vésico-ureteral $(25,0 \%)$, válvula de uretra posterior (18,7\%) e outros (21,9\%). Todos foram submetidos a avaliação urológica com uretrocistografia miccional e estudo urodinâmico completo (cistometria, eletromiografia, urofluxometria e medida do volume residual), além de cintilografia renal ao DMSA e outros exames de trato urinário conforme indicação.

\section{RESULTADOS}

Foram realizados 35 transplantes em 32 pacientes com uropatia, $30(85,7 \%)$ de doador vivo relacionado, com mediana de idade no transplante de 12,2 anos (limites 4,7 a 22,8), mediana do tempo de espera de 17,0 meses ( 0 a 104,0), 13 (37,1\%) com ampliação vesical $(\mathrm{p}=0,055)$ : estômago $(15,4 \%)$, estômago /intestino $(7,7 \%)$, íleo (7,7\%), sigmóide (53,8\%) e ureter $(15,4 \%)$. Entre os 35 transplantes realizados foram diagnosticados 10 episódios de rejeição aguda $(28,6 \%), 11$ de rejeição crônica $(31,4 \%)$ e 16 de infecção de trato urinário (ITU) (45,7\%). Houve 10 (28,6\%) perdas de enxerto e um paciente $(3,1 \%)$ evolui para óbito (Figura 1$)$.

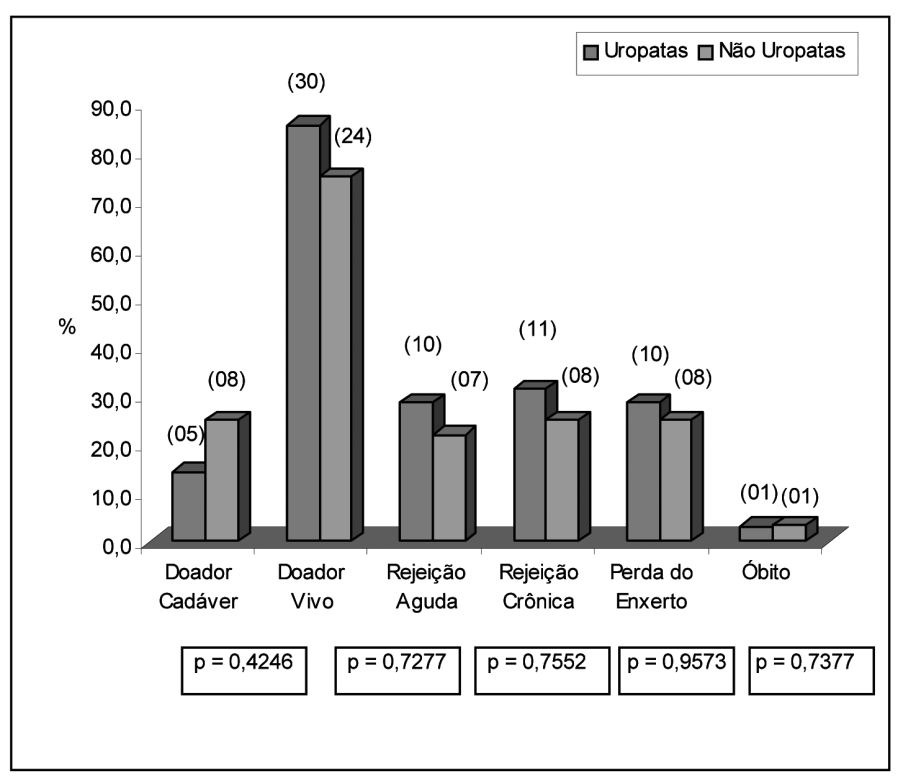

Figura 1 - tipo de doador e evolução pós-transplante
No grupo controle - 30 pacientes sem doença urológica - foram realizados 32 transplantes renais, $24(75,0 \%)$ de doador vivo relacionado, com mediana de idade no transplante em 11,1 anos (limites 4,6 a 19,1), mediana do tempo de espera em 23,5 meses $(8,0$ a 84,0$)$. Entre os 30 transplantes realizados foram diagnosticados 7 episódios de rejeição aguda $(21,9 \%)$ e 8 de rejeição crônica $(25,0 \%)$ e nenhum episódio de infecção urinária. Houve $8(25,0 \%)$ perdas de enxerto. Um paciente (3,3\%) evoluiu para óbito (Tabela 1, Figura 1).

Tabela 1 - UROPATAS: procedimentos pré e pós-transplante e evolução

\begin{tabular}{|c|c|c|c|}
\hline DADOS & $\begin{array}{c}\text { № UROPATAS } \\
(n=35)\end{array}$ & $\%$ & VALOR $p$ \\
\hline Procedimentos pré-transplante & & & $<0,0001$ \\
\hline - Unilateral & 03 & 8,6 & \\
\hline - Bilateral & 29 & 82,8 & \\
\hline Ampliação Vesical & 13 & & 0,055 \\
\hline • Estômago & 02 & 15,4 & \\
\hline - Estômago/Intestino & 01 & 7,7 & \\
\hline • Íleo & 01 & 7,7 & \\
\hline - Sigmóide & 07 & 53,8 & \\
\hline - Ureter & 02 & 15,4 & \\
\hline Cateterismo do Conduto & 16 & 45,7 & 0,631 \\
\hline \multicolumn{4}{|l|}{ Procedimentos pós-transplante } \\
\hline - Cateterismo Uretral & 03 & 8,6 & $<0,0001$ \\
\hline - Cateterismo em Conduto & 15 & 42,9 & 0,343 \\
\hline - Oxibutinina & 16 & 45,7 & 0,631 \\
\hline - Antibiótico Profilático & 22 & 62,9 & 0,055 \\
\hline \multicolumn{4}{|l|}{ Evolução } \\
\hline - Refluxo Vésico-Ureteral & 30 & 85,7 & $<0,0001$ \\
\hline - Cicatriz Renal & 32 & 91,4 & $<0,0001$ \\
\hline - Litíase & 33 & 94,3 & $<0,0001$ \\
\hline - Infecção do Trato Urinário & 19 & 45,7 & $<0,00003$ \\
\hline - $<5$ episódios & 08 & 42,1 & \\
\hline - > 5 episódios & 11 & 57,9 & \\
\hline - Hidronefrose & 26 & 74,3 & $<0,0001$ \\
\hline
\end{tabular}

Não houve diferença significativa entre o grupo dos uropatas e o controle exceto quanto ao tempo de espera para o transplante $(p=0,006)$.

No grupo de uropatas houve $3(8,6 \%)$ nefrectomias unilaterais e 29 (82,8\%) bilaterais, 13 (37,1\%) ampliações vesicais e 16 (45,7\%) condutos para cateterismo. Antes do transplante esses pacientes foram tratados com cateterismo intermitente $(42,9 \%)$, oxibutinina (45,7\%), quimioprofilaxia para infecção do trato urinário (62,9\%). A incidência de complicações pós-transplante foi de $14,3 \%$ para refluxo vésico-ureteral, 8,6\% para cicatrizes renais, 5,7\% para litíase, $11,4 \%$ para linfocele e $25,7 \%$ para hidronefrose (Tabela 1 ).

Quando comparou-se desfechos clínicos entre pacientes com e sem ampliação vesical não houve diferença significativa na incidência de litíase e refluxo vésico-ureteral, nas medianas do clearance de creatinina e do tempo de seguimento. A diferença na incidência de infecção do trato urinário e de hidronefrose pós-transplante foi significativa (Tabela 2, Figura 2). 


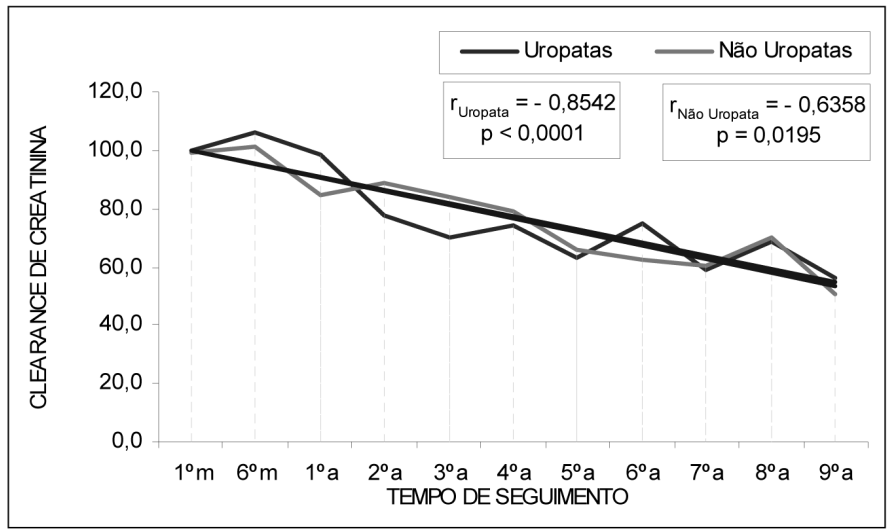

Figura 2 - uropatas e não-uropatas: função do enxerto do enxerto

Doze $(37,5 \%)$ pacientes uropatas foram submetidos a 13 ampliações vesicais e a comparação entre o estudo urodinâmico pré-ampliação e pré-transplante e pré e pós-transplante demonstrou diferença significativa na mediana da capacidade vesical: 160,0 versus 280,0 $\mathrm{ml}(\mathrm{p}<0,0001)$ e 280 versus 480,0 $\mathrm{ml}(\mathrm{p}=0,009)$, respectivamente. A diferença na complacência vesical foi significativa quando comparou-se a mediana pré-ampliação e pré-transplante: 4,0 versus $13,8 \mathrm{kpa}(\mathrm{p}=0,002)$. Não houve diferença significativa na comparação das medianas no pré e pós-transplante e do volume residual

\section{DISCUSSÃO}

A evolução do transplante renal em crianças com insuficiência renal crônica secundária a uropatia é semelhante a das não-uropatas desde que se corrijam as anomalias do trato urinário e se garanta uma boa função vesical. ${ }^{1-3}$ Neste estudo a investigação, diagnóstico e correção cirúrgica das alterações urinárias congênitas e tratamento clínico das disfunções vesicais, integrados com o transplante, resultaram em incidência de desfecho semelhante entre uropatas e não-uropatas, em especial os relacionados com função e sobrevida do enxerto e sobrevida dos pacientes. Incidências essas similares às do relatório 1987-2001 do North American Pediatric Renal Transplant Cooperative Study(4). O objetivo principal do tratamento é conseguir um armazenamento adequado, esvaziamento a baixa pressão $-<40$ $\mathrm{mm} \mathrm{Hg}$-, sem resíduo e com continência, e para isto deve-se utilizar a estratégia mais simples e efetiva possível, visto que não há tratamento perfeito. ${ }^{3}$ A abordagem do trato urinário, quando não contempla um futuro transplante renal, resulta em muitas e desnecessárias cirurgias que dificultam o procedimento do transplante, particularmente em crianças pequenas, em quem a dissecação da aorta abdominal e da veia cava é prejudicada pela presença de tecido fibrótico. A retirada precoce de um rim com megaureter significa privar a criança do melhor tecido disponível para uma futura ampliação vesical prétransplante. ${ }^{1,2} \mathrm{O}$ planejamento cirúrgico é fundamental.
Tabela 2 - uropatas: pacientes uropatas com e sem ampliação vesical: evolução pós-transplante

\begin{tabular}{|c|c|c|c|c|c|c|c|}
\hline \multirow[t]{2}{*}{ DADOS } & \multicolumn{2}{|c|}{$\begin{array}{l}\text { COM } \\
\text { AMPLAÇÃO } \\
(n=12) \\
\end{array}$} & \multicolumn{2}{|c|}{$\begin{array}{c}\text { SEM } \\
\text { AMPLIAÇÃO } \\
(n=22)\end{array}$} & \multicolumn{2}{|c|}{$\begin{array}{l}\text { TOTAL } \\
(n=34)\end{array}$} & \multirow[t]{2}{*}{ VALOR $p$} \\
\hline & & & & & $\mathrm{N}$ & $\%$ & \\
\hline $\begin{array}{l}\text { Infecção do Trato Urinário } \\
\text { : Não } \\
\text { Sim }\end{array}$ & $\begin{array}{l}01 \\
11\end{array}$ & $\begin{array}{c}8,3 \\
91,7\end{array}$ & $\begin{array}{l}14 \\
08\end{array}$ & $\begin{array}{l}63,6 \\
36,4\end{array}$ & $\begin{array}{l}15 \\
19\end{array}$ & $\begin{array}{l}44,1 \\
55,9\end{array}$ & (1) 0,0061 \\
\hline $\begin{array}{l}\text { Litíase } \\
\text { • Não } \\
\text { Sim }\end{array}$ & $\begin{array}{l}11 \\
01\end{array}$ & $\begin{array}{c}91,7 \\
8,3\end{array}$ & $\begin{array}{l}21 \\
01\end{array}$ & $\begin{array}{c}95,5 \\
4,5\end{array}$ & $\begin{array}{l}32 \\
02\end{array}$ & $\begin{array}{c}94,1 \\
5,9\end{array}$ & (2) 0,5882 \\
\hline $\begin{array}{l}\text { RVU pós-transplante } \\
\text { - Não } \\
\text { - Sim }\end{array}$ & $\begin{array}{l}10 \\
02\end{array}$ & $\begin{array}{l}83,3 \\
16,7\end{array}$ & $\begin{array}{l}19 \\
03\end{array}$ & $\begin{array}{l}86,4 \\
13,6\end{array}$ & $\begin{array}{l}29 \\
05\end{array}$ & $\begin{array}{l}85,3 \\
14,7\end{array}$ & (2) 0,5899 \\
\hline $\begin{array}{l}\text { Hidronefrose pós-transplante } \\
\text { : Não } \\
\text { Sim }\end{array}$ & $\begin{array}{l}05 \\
07\end{array}$ & $\begin{array}{l}41,7 \\
58,3\end{array}$ & $\begin{array}{l}20 \\
02\end{array}$ & $\begin{array}{c}90,9 \\
9,1\end{array}$ & $\begin{array}{l}25 \\
09\end{array}$ & $\begin{array}{l}73,5 \\
26,5\end{array}$ & (2) 0,0037 \\
\hline $\begin{array}{l}\text { Creatinina } \\
\text { - Média } \pm \text { desvio padrão } \\
\text { - Minima e máxima } \\
\text { - Mediana }\end{array}$ & $\begin{array}{l}3,1 \\
0,6\end{array}$ & $\begin{array}{r}2,5 \\
6,8 \\
7\end{array}$ & $\begin{array}{l}2,9 \\
0,6\end{array}$ & $\begin{array}{l} \pm 2,8 \\
9,5 \\
1,8\end{array}$ & $\begin{array}{l}3,0= \\
0,6 \\
\end{array}$ & $\begin{array}{l}+2,7 \\
9,5 \\
8\end{array}$ & ${ }^{(3)} 0,842$ \\
\hline $\begin{array}{l}\text { Clearance creatinina (fórmula } \\
\text { de Schwartz) } \\
\text { : Média } \pm \text { desvio padrão } \\
\text { - Mínima e máxima } \\
\text { Mediana }\end{array}$ & $\begin{array}{r}61,2 \\
12,0 \\
5\end{array}$ & $\begin{array}{l}46,8 \\
147,0 \\
, 5\end{array}$ & $\begin{array}{r}55,5 \\
9,0 \\
5\end{array}$ & $\begin{array}{r} \pm 30,8 \\
131,0 \\
8,0\end{array}$ & $\begin{array}{r}57,5 \\
9,0 \\
57\end{array}$ & $\begin{array}{r}36,3 \\
147,0 \\
7,5\end{array}$ & ${ }^{(3)} 0,842$ \\
\hline $\begin{array}{l}\text { Tempo de seguimento (anos) } \\
\text { : Média } \pm \text { desvio padrão } \\
\text { : Minima e máxima }\end{array}$ & ${ }_{0,0}^{3,5}{ }_{3}^{ \pm}$ & $2^{3,2} 9,7$ & $2,9=$ & $=3,3$ & $\begin{array}{r}3,7 \pm \\
0,0 \\
3\end{array}$ & ${ }^{3,2} 6^{11,3}$ & ${ }^{(3)} 0,943$ \\
\hline
\end{tabular}

A instabilidade do detrusor responde ao uso de anticolinérgicos como a oxibutinina. ${ }^{1}$ A irrigação vesical através do cateterismo intermitente pode ser necessária para manter ou aumentar a capacidade e complacência da bexiga nativa ou ampliada no pré-transplante de pacientes oligúricos ou anúricos. $\mathrm{O}$ estudo urodinâmico na presença de uma diurese razoável permite que, futuramente, se reconheçam as bexigas de desuso, que não necessitam de ampliação com recuperação da capacidade e complacência vesical após o transplante. A ampliação vesical exige uma seleção cuidadosa de pacientes devido à necessidade de adesão ao cateterismo intermitente e a possíveis complicações como litíase, urosepse e risco de malignização. Virtualmente todos os segmentos do trato gastrointestinal já foram utilizados para ampliação vesical e cada um apresenta vantagens e riscos. ${ }^{1}$ Atualmente o ureter dilatado é mais utilizado.

\section{CONCLUSÃO}

Este estudo demonstra que o transplante renal em uropatas é seguro e efetivo e depende de avaliação urológica detalhada prétransplante, da correção das anormalidades e disfunções do trato urinário inferior e, também, de acompanhamento diferenciado no pós-transplante, que possibilite o diagnóstico e o tratamento precoce das possíveis complicações e incentive a aderência a procedimentos específicos como cateterismo vesical, quimioprofilaxia e lavagem vesical, sempre que necessário. Garante-se assim a função e sobrevida do enxerto e uma menor morbidade para o paciente.

\section{ABSTRACT}

Introduction: Obstructive uropathy is a common etiology of end stage renal disease in infancy and childhood. A clinical-surgical protocol that aims for reconstruction of the urinary tract before transplantation has been considered fundamental in the graft prognosis. Objective: to compare graft function and survival in patients with and without uropathies and to describe outcomes in patients with 
augmented bladder. Methods: we studied 62 kidney transplanted patients from 1990 to 2000, in "Hospital Pequeno Principe de Curitiba", Parana, Brazil, with more than 6 months of follow-up; 32 (51,6\%) with uropathy. All have been submitted to urological evaluation before and after transplantation. Results: fifty eight percent of the 62 patients were male, age of 12,1 \pm 4 years (mean \pm standard deviation). Comparing uropathic and non-uropathic patients, there was no significant difference in relation to sex, age, mortality, living or cadaver donor, compatibility, acute rejection, chronic graft nephropathy, graft loss and creatinine clearance during the follow-up. Urinary tract infection ocurred only in the group with uropathies. Among those with urological disorder, previous unilateral nephrectomy was done in $8,6 \%$ and bilateral in $82,8 \%$, bladder augmentation was done in $37,1 \%$ and continent conduit in $45,7 \%$. Urodynamic studies showed a significant difference in the bladder capacity and compliance before and after augmentation and after transplant. Those who had undergone augmentation did not have more vesicoureteral reflux or lithiasis, but had more urinary tract infection and hydronephrosis. Conclusion: specific pre-transplantation and follow-up clinical-surgical protocols for children with obstructive uropathy was related with a favorable evolution and with good renal graft prognosis.

Key words: Kidney, Live donor, Renal transplantation, Nephrectomy, Laparoscopy.

\section{REFERÊNCIAS}

01. Salvatierra O Jr, Alfrey E, Tanney DC et al. Superior outcomes in pediatric renal transplantation. Arch Surg 1997; 132: 842-849.

02. Churchill BM, Jayanthi RV, McLorie GA, Khoury AE. Pediatric renal transplantation into the abnormal urinary tract. Pediatr Nephrol 1996; 10: 113-120.
03. Salvatierra O Jr, Alexander SR, Krensky AM. Pediatric transplantation at Stanford. Pediatr Transplantation 2000; 4: 118-122.

04. Smith, Jodi M., Ho, P. L. (M.) \& McDonald, Ruth A. Renal transplant outcomes in adolescents: A report of the North American Pediatric Renal Transplant Cooperative Study. Pediatric Transplantation 2002; 6: 493-499 